18 Schinzel A, D'Apuzzo V. Anophthalmia in a retarded girl with partial trisomy $4 \mathrm{p}$ and 22 following a maternal translocation, rcp $(4 ; 22)(\mathrm{pl} 15.2 ; \mathrm{q} 11.2)$. Ophthalmol Paediat Genet 1990; 11: $139-42$.

19 Herva $R$, von Wendt L. De novo trisomy 4pter $\rightarrow \mathrm{q} 21$. Hum Genet 1978; 41: 225-30.

20 Wilson MG, Towner JW, Coffin GS, Forsman I. Inherited pericentric inversion of chromosome No. 4. Am f Hum Genet 1970; 22: 679-90.

21 Wilson MG, Towner JW, Coffin GS, Ebbin AJ, Siris E, Brager $P$. Genetic and clinical studies in 13 patients with the Wolf-
Hirschhorn syndrome [del(4p)]. Hum Genet 1981; 59: 297307.

22 Narahara K, Himoto Y, Yokoyama Y, Kasai R, Hata A, Kikkawa $\mathrm{K}$, et al. The critical monosomic segment involved in $4 \mathrm{p}$ - syndrome: a high-resolution banding study on five inherited cases. F pn f Hum Genet 1984; 29: 403-13.

23 Mayer UM, Bialasiewicz AA. Ocular findings in a 4p-deletion Mayer UM, Bialasiewicz AA. Ocular findings in a 4p- deletion
syndrome (Wolf-Hirschhorn). Ophthalmol Paediat Genet 1989; 10: 69-72.

24 Mortimer JG, Chewings WE, Gardner RJM. A further report on a kindred with cases of $4 \mathrm{p}$ trisomy and monosomy. Hum Hered 1980; 30: 58-61.

\title{
Meningitis as a complication of dacryocystorhinostomy
}

\author{
Itzchak Beiran, Josef Pikkel, Michael Gilboa, Benjamin Miller
}

\begin{abstract}
A blocked lacrimal drainage system may cause much inconvenience because of epiphora and recurrent infections. Lacrimal block can give way to serious complications such as recurrent dacryocystitis (acute or chronic) and orbital cellulitis, which is a potentially life threatening condition. ${ }^{1}$ The ultimate solution for a blocked drainage system is surgical opening. Dacryocystorhinostomy is a commonly used procedure for relieving lacrimal drainage obstruction. Among the possible complications of this operation are inadequate drainage because of intranasal pathology, contracted sac, or postoperative closure and intraoperative bleeding either as a result of incision of the nasal mucosa or, in more severe bleeding, because of laceration of the anterior ethmoidal artery in anteriorly placed ethmoidal air cells. No mention of postoperative infectious complications in remote organs has been made in English language reports. ${ }^{1}$ We feel it is of interest, therefore, to report a case in which bacterial meningitis developed in an otherwise healthy girl 1 day after dacryocystorhinostomy.
\end{abstract}

\footnotetext{
Department of

Ophthalmology,

Rambam Medical Center and the Bruce Rappaport Faculty of Medicine, Technion-Israel Institute of Technology, Haifa, Israel

I Beiran

J Pikkel

M Gilboa

B Miller

Correspondence to Benjamin Miller, MD, Department of Ophthalmology, Rambam Medical Center, PO Box 9602, 31096 Haifa, Israel. Accepted for publication 24 November 1993
}

\begin{abstract}
Case report
A 9-year-old girl was admitted to our department for dacryocystorhinostomy because of right nasolacrimal obstruction. She had a history of recurrent dacryocystitis and epiphora since birth and had not been relieved by two attempts at tubing and probing. On admission, no history of upper respiratory tract infection in the child or in her close neighbourhood was reported, nor was any other complaint reparted. On physical examination, the child looked healthy; body temperature was $36 \cdot 7^{\circ} \mathrm{C}$, and no pathology was revealed except epiphora. The lacrimal sac was neither enlarged nor tender and showed no signs of acute infection.
\end{abstract}

The following day the girl underwent a dacryocystorhinostomy. Surgical incision was made about $8 \mathrm{~mm}$ medial to the medial commissure, beginning at the level of the medial canthal tendon and extending downward some $15 \mathrm{~mm}$. The skin incision and angular vein were put on haemostatic suture. The ipsilateral nostril was packed with an ENT tampon saturated with $10 \%$ cocaine and 1:1000 adrenaline solution in the region of the attachment of the anterior end of the middle turbinate.

The subcutaneous tissue was separated with Freer elevators down to the bone, and the periosteum was elevated. The medial canthal tendon was put on suture and cut down to the bone. The periosteum was elevated on both sides of the incision and over the anterior lacrimal crest downward into the nasolacrimal canal and backward to the posterior lacrimal crest. The bone of the anterior lacrimal crest and wall of the lacrimal fossa were removed with a small trephine and the nasal mucosa carefully separated from the bony margins. The opening was enlarged with a flat nosed Kerrison punch. The bony medial half of the nasolacrimal canal was removed. The nasal mucoperiosteum was elevated from the canal down to the inferior turbinate. The sac was opened by a pointed Bard-Parker blade through both the periosteal and mucosal walls, and a similar incision was made in the nasal mucoperiosteum adjacent and parallel to the one in the tear sac.

Flaps were sutured using $6 / 0$ plain gut after washing out the anastomosis with an antibiotic solution (amoxicillin). The periosteum was closed with a running $6 / 0$ plain gut suture, and the subcutaneous tissue with a similar one. The skin was closed with a running mattress $6 / 0$ nylon suture. Nasal packing was changed with a sterile piece of gauze.

During the operation body temperature was 
monitored and found to be normal. In the first hours postoperatively the patient regained full consciousness, acted normally, and reported only a mild degree of pain in the operated area, with no other complaints.

The morning following the operation (about 20 hours after surgery) the patient complained of severe headache and displayed stuporous behaviour. Physical examination revealed a body temperature of $38.9^{\circ} \mathrm{C}$ and overt nuchal rigidity. Lumbar puncture results were typical of bacterial meningitis (white blood count showed 800 polymorphonuclear white blood cells and glucose $30 \mathrm{mg} / \mathrm{dl}$ ). Bacteriological study revealed diplococci on direct examination. Growth in culture medium after 24 hours showed those organisms to be Streptococcus pneumoniae.

The patient was treated by antibiotic therapy (ceftriaxone intravenously, $2 \mathrm{~g} /$ day in one dose for 10 days, along with dexamethasone $1.2 \mathrm{~g} /$ day, in four divided doses in the first 4 days of treatment). Following 2 days of treatment, the patient had no headache and had regained full consciousness; body temperature was $36 \cdot 8^{\circ} \mathrm{C}$; no nuchal rigidity was present, and the rest of the physical examination was normal (including patient lacrimal drainage system). At discharge from the hospital, physical examination was within normal limits.

\section{Comment}

Dacrycystorhinostomy is frequently performed on patients with a long standing history of obstructed lacrimal drainage system and recurrent dacryocystitis, either acute or chronic. It can be considered, therefore, to be taking place in contaminated surroundings. The most prevalent pathogens playing a role in lacrimal sac infections are Streptococcus pneumoniae, Staphylococcus, and diphtheroids. Other reported pathogens include Klebsiella, Haemophilus, and Pseudomonas. Meningitis has not been mentioned in English language reports as a possible complication of otherwise uncomplicated dacryocystorhinostomy.

The source of pathogens causing meningitis is either haematological spread or local invasion from adjacent infected tissues. ${ }^{2}$ In the case of dacryocystorhinostomy, consideration must be given to both possible routes. Pneumococcal meningitis is usually preceded by several days of upper respiratory or gastrointestinal symptoms. The fact that no such symptoms were present in this patient, gave further support to the sug gested connection between the operation and the appearance of meningitis.

In the presented case, the haematological route seems more likely. The infection took place within $\mathbf{2 4}$ hours; this rapid appearance is typical of blood borne infection, as local invasion is expected to take longer to develop. Also, the meningitis appeared with no local ocular or periocular signs; local invasion would be expected to cause a violent reaction at the site of the invading infection in the form of periorbital cellulitis or ethmoiditis. Finally, only a single pathogen was isolated from the cerebrospinal fluid. In local invasion, usually more than one pathogen gains access to the site of infection, so that mixed culture is grown on culture medium.

In a previous report on epiphora resulting from cerebrospinal fluid leak, meningitis was observed after dacryocystorhinostomy as a result of surgical manipulations. ${ }^{3}$ That case was basically different from the one presented here, as it dealt with a patient who had suffered naso-orbital injury in a road accident 2 years before the operation. As a result of that injury, the patient's lacrimal drainage system became obstructed. The same trauma caused a fracture in the posteromedial roof of the orbit with encephalocele into it. Because of this fracture, no anatomical barrier existed between brain meninges and the orbit. Even though direct anatomical access existed between the orbit and the meninges, it took 9 days for meningitis to appear, and even then it was preceded 2 days earlier by local signs of overt periorbital cellulitis.

As no bacteriological investigation was reported in that case, it is impossible to propose causative organisms as another possible difference between that case and ours. That case demonstrated classic signs of meningitis caused by local invasion and differed in all significant characteristic mechanisms of disease from our case (which was typical of haematogenous spread).

Anatomical dissection performed on cadavers from an elderly population showed the distance between the point located $10 \mathrm{~mm}$ posterior to the medial ligament and the anterior cranial fossa to be $8.3 \mathrm{~mm}$ on average. ${ }^{4}$ In $21.4 \%$ of the specimens the distance was $3 \mathrm{~mm}$ or less. The conclusion of that report was that enlarging the posterior nasal window more than $3 \mathrm{~mm}$ may cause a bony opening in the floor of the anterior cranial fossa. ${ }^{4}$ Apparently, if such an anatomical defect gives way to meningitis, it should have the characteristics of a local invasive infection.

As the case presented here displayed the characteristics of blood borne infection and none of a locally invasive one (as discussed above), injured anterior cranial fossa bony wall is not likely to have played a part in the development of meningitis in the presented patient.

No definite proof exists as to a connection between the dacryocystorhinostomy and the meningitis in this case. However, the appearance of meningitis in a formerly completely healthy child with no upper respiratory tract infection and no environmental exposure 20 hours after dacryocystorhinostomy strongly suggests a causative connection between the two.

We believe that this case adds a meaningful possible complication of dacryocystorhinostomy to those already reported and should alert the medical team to the special attention needed to detect possible early signs of meningitis. Awareness of this life threatening complication is the key to early detection and treatment critical for successful therapy.

$1 \mathrm{McCord}$ CD Jr. The lacrimal drainage system. In: Tasman W, Jaeger EA, eds. Duane's clinical ophthalmology. Vol 4. Philadelphia: Lippincott, 1989: 8-25.

2 Kliegman RM, Behrman RE. Acute bacterial meningiti beyond the neonatal period. In: Behrman RE, Kliegman RM Nelson WE, Vaughan VC, eds. Nelson's textbook of pediatrics. Philadelphia: Saunders, 1992: 683-91.

3 Dryden RM, Wulc AE. Pseudo-epiphora from cerebrospina fluid leak: case report. $B r \mathcal{F}$ Ophthalmol 1986; 70:570-4.

4 Kurihashi K, Yamashita A. Anatomical consideration for dacryocystorhinostomy. Ophthalmologica 1991; 203: 1-7. 